\title{
A 9T FinFET SRAM cell for ultra-low power application in the subthreshold regime
}

\author{
Shilpi Birla ${ }^{1}$, Neha Singh ${ }^{2}$, Neeraj K. Shukla ${ }^{3}$, Sidharth Sharma ${ }^{4}$ \\ ${ }^{1,2}$ Department of Electronics and Communication Engineering, Manipal University Jaipur, India \\ ${ }^{3}$ Department of Electrical Engineering, King Khalid University, Abha, Saudi Arabia \\ ${ }^{4}$ ServiceNow, $1^{\text {st }}$ Floor, The Sharp Building, 10-12 Hogan PI, Dublin, Ireland
}

\begin{tabular}{l} 
Article Info \\
\hline Article history: \\
Received Sep 7, 2020 \\
Revised Mar 2, 2021 \\
Accepted Oct 25, 2021 \\
\hline Keywords: \\
FinFET \\
Nano-scaled devices \\
RSNM \\
Subthreshold \\
WSNM
\end{tabular}

\begin{abstract}
Due to the scaling of the CMOS, the limitations of these devices raised the need for alternative nano-devices. Various devices are proposed like FinFET, TFET, CNTFET. Among these, the FinFET emerges as one of the promising devices which can replace the CMOS due to its low leakage in the nanometer regime. The electronics devices are nowadays more compact and efficient in terms of battery consumption. The CMOS SRAMs have been replaced by the FinFET SRAMs due to the scaling limitations of the CMOS. Two FinFET SRAM cells have been which power efficient are and having high stability. Performance comparison of these cells has been done to analyze the leakage power and the static noise margins. The simulation of the cells is done at $20 \mathrm{~nm}$ FinFET technology. It has been analyzed that the write margin of improved 9T SRAM cell achieves an improvement of 1.49x. The read margin is also showing a drastic improvement over the existing cells which has been compared in the paper. The hold margin was found to be better in the case of the proposed SRAM cell at $0.4 \mathrm{~V}$. The gate length has been varied to find the effect on read margin with gate length.
\end{abstract}

This is an open access article under the CC BY-SA license.

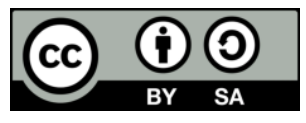

\section{Corresponding Author:}

Shilpi Birla

Department of Electronics and Communication Engineering

Manipal University Jaipur

Dehmi Kalan, Jaipur, India

Email: 1sntl@ccu.edu.tw

\section{INTRODUCTION}

Miniaturization has become the trend for electronic gadgets. The market trend is towards handy portable and compact devices. Almost all the electronics devices have the involvement of CMOS and scaling of the CMOS has made the miniaturization dream possible. All smart gadgets are nowadays coming in small compact sizes. These devices have various circuits but the one which is common to all is processor and memory. When we think of a handheld device at the same time, we can realize that the memory size would also be reduced. The speed of the devices should also be fast, so memories like SRAM started gaining popularity.

Scaling of the sizes is possible because of the reduction of the size of CMOS, this size reduction has come up with certain issues like power and stability of the devices. Both the issues of power and stability are important performance matrix of the SRAM Memories. Several issues like high power consumption, leakage current, stability, process variations. have been raised and addressed by the researchers. The reduction in the supply voltage which is necessary due to the scaling of the device size also resulted in the reduction of the threshold voltage of the CMOS devices which was one of the major concerns. So many solutions have been proposed among them the subthreshold operation [1] was one of the solutions, where the device works below 
the threshold voltage, but it affected the 6T SRAM stability issues. To address the limitations the conventional 6T SRAM cell was then replaced by other versions of SRAM cells like 7T, 8T, 9T, 10T SRAM cells for enhancing stability criteria and reduce the power.

\section{LITERATURE REVIEW}

CMOS scaling has now come to a limit and alternate solutions for silicon or CMOS devices have come up like TFET, FinFETs, CNTs. FinFET device seems to be a promising device and can be one of the most promising candidates for low power emerging electronics circuits. FinFETs can work at low medium frequency operations at lower voltages. FinFET has higher threshold voltages and it has overcome the short channel effect of CMOS, so this can be another good choice for SRAM memories [2], [3]. Memories using FinFET has been proposed and accepted largely due to the benefit of small size and low leakage over CMOS. After Intel's recent announcement that FinFET will be its basic CMOS device beginning at the 22 nm node, the development of nanoscale FinFET has increased [4]. The number of gates in FinFET can be two, or even three. The quasi-planar structure, and the uncommon processing thereby implied, can thus be effectively traded off. FinFETs are being fabricated on the bulk and silicon on insulator (SOI) wafers. In bulk FinFETs, all fins used a common Si substrate while in SOI fins which are physically isolated.

Based on the gates FinFETs can be classified as shorted gate and independent gate FinFETs. A FinFET whose gates are independently controlled is described as an independent-gate (IG) FinFET, whereas in shorted gate as the name depicts both the gates of the FinFET are being shorted. Tied gate FinFET is the other name of the SG FinFET. Threshold voltage can be modulated by biasing the gates of IG FinFET which can be useful for reducing the leakage power. SG FinFETs are the fastest as they provide better control of the channel and improved drive strength [5], [6].

To enhance the stability and reduce the power various SRAMs have been proposed from time to time by researchers. SRAM has been classified as single port \& dual port (multiport), symmetric \& asymmetric SRAMs. A novel 9T SRM cell using CMOS has been proposed in [7]. Pal and Islam [8] 8T SRAM has been proposed for enhanced stability and low leakage. Some FinFET SRAMs have also been proposed for improvements in terms of stability and leakage. Gupta et al. [9] author proposed SRAM with pass gate feedback. To increase robustness in FinFET SRAM author has used pass p-type access transistor [10]. 8T FinFET SRAM cell has been proposed with improved read and write margins in [11]. Birla [12] subthreshold FinFET SRAM has been proposed for low power applications. Robust 6T FinFET SRAM cell has been studied in [13]. In this work a 9T SRAM using FinFET has been compared with 9T improved FinFET SRAM in which 9 transistors have been used to improve the leakage and the stability of the cell. The 10T SRAM FinFET SRAM is proposed with high stability and read margin [14]. Feedback mechanism using Schmitt trigger-based SRAMs are proposed in [15], [16]. Dual port SRAM for imptoved stability has been proposed in [17]. FinFET SRAM with minimum variatins and improved stability has been proposed in [18]. Finfet SRM with asymmetrical bitlines has been proposed in [19].

\section{PROPOSED WORK}

In this paper, an SRAM cell consisting of nine FinFET transistors has been proposed. Due to the limitation of the scaling down of CMOS device FinFET has been used to improve the overall performance which includes efficiency, power, and area. Various FinFET SRAM cells have been proposed by the designers which are low leakage and having improved static noise margins. These cells are proposed for an improvement in the leakage current and stability of the cell.

Figure 1 shows a conventional 6T transistor using FinFET. Here, two-bit lines: One is named as BL and the other as BLB is used. The one-word line named WL is also used to evaluate the working of the FinFET SRAM cell. The structure of the cells looks like a basic 6T cell, the only thing with 3 extra transistors. The main problem with the conventional 6T transistors as read disturb. However, due to device scaling, voltage is scaled down which results in decreased noise margins. This lowers the stability of the 6T SRAM cell. Figure 2 and Figure 3 shows the two proposed cells namely, 9T FinFET SRAM and 9T improved FinFET SRAM, the cells have a structure similar to 8T SRAM with one extra transistor deployed to enhance the performance of the cell.

\subsection{T FinFET SRAM cell}

Figure 2 shows the projected 9T SRAM cell using FinFET consisting of nine N-type FinFET transistors. The extra transistors used in this circuit from the conventional circuit helps in reducing the leakage current and the SRAM removes the limitation of the single port SRAM cell as it is a dual-port SRAM cell. The write operation is performed when the word line (WL) is kept at a high voltage which turns the access FinFET transistors of the cell ON. Since transistor M9 is connected to WL which is high so M9 will also be 
high. Q and QB are the storage nodes assuming that initially Q stores logic 1 and QB will store logic 0 . To write logic 0 at node Q we need to keep BL at logic 0 and BLB at Vdd. Node Q will discharge through M5 by BL and voltage at node QB starts increasing toward Vdd through M6 by BLB Voltage stored at node Q will start discharging through M7 to the ground. While node QB starts charging as BLB is kept at Vdd through the access transistor. Node Q has the other two paths to discharge through M4 and M9 and also through M8. So data is written successfully. Similarly, for writing logic 1 at Q and QB at logic 0 keep the BL at Vdd and BLB at logic 0 .

While reading, i.e., the read operation, bit-lines (BL and BLB) are precharged to Vdd and then keeping the WL to Vdd. Transistor M9 will be ON during the read and write operation. If node Q is at logic 1, transistor M4 will be ON and access transistor M5 will be ON. BL will be discharged through M5 and either through path M4 and M9 or M8 and ground. Similarly, if Q is at 0, BL will be discharged through M5 which is ON followed by M7 and ground. So, depending on the content of the storage nodes M3/M4 and M7/M8 will be ON and helps in discharging the BL. Hold operation is done by lowering the word line to GND as done in conventional 6T transistors. If WL will be assigned logic 0 the extra transistor M9 will also be Off, helping in reducing the leakage power.

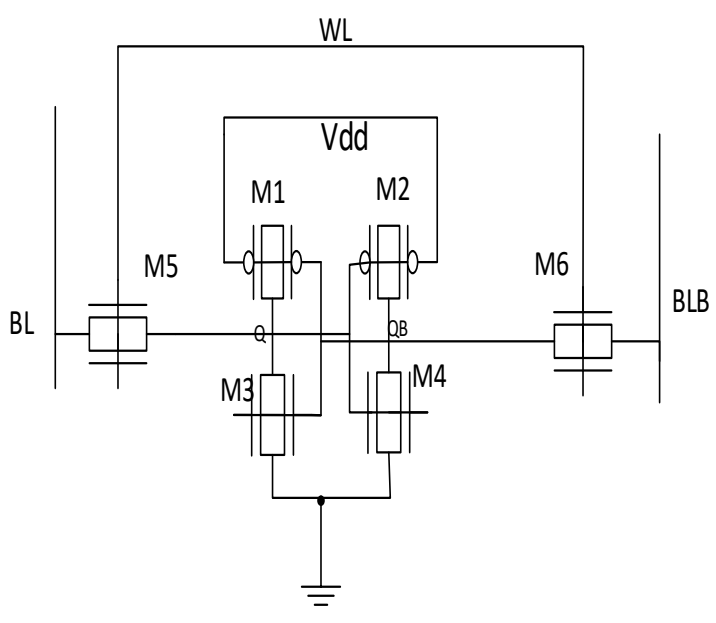

Figure 1. Conventional 6T SRAM FinFET cell

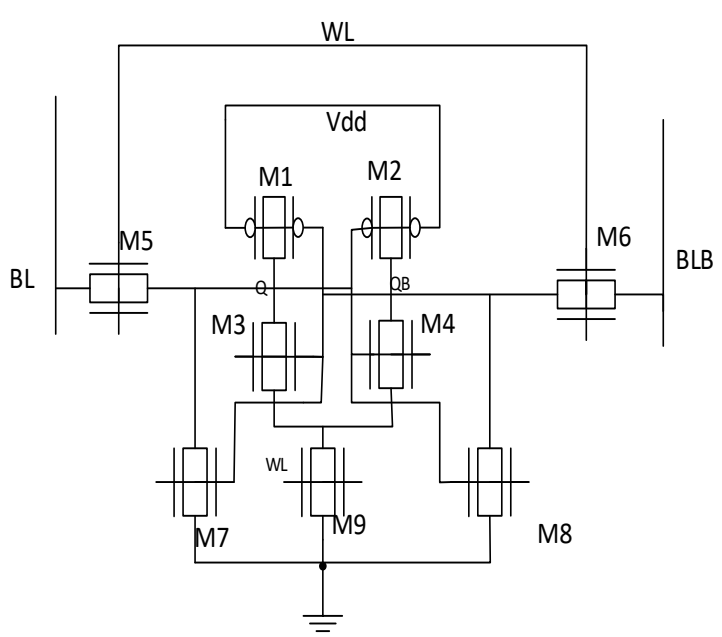

Figure 2. 9T SRAM FinFET cell

\subsection{T FinFET improved SRAM cell}

The improved version of the proposed 9T SRAM cell is similar in structure and working as to the 9T SRAM using FinFET. The improved version uses an extra transistor used is PMOS. This PMOS is being utilized in place of the NMOS in the 9T SRAM cell. Here, the extra transistor is PMOS which is controlled by a Cnt signal. The WL should be high, the bit lines are high according to the data which is to be written into the memory cell, Let's assume that the data to be written is logic 1, in this case, the BL should be kept at high voltage and BLB should be at low voltage. The only difference in this proposed cell is that here we have a control signal (Cnt) which remains logic "0" during read and write operations. This in turn on the PMOS which helps in reducing leakage current. The working of the cell is similar to the 9T FinFET SRAM cell as discussed in section 2 .

\section{PERFORMANCE ANALYSIS OF THE CELL}

Various performance parameters of the cells are being considered in this paper like leakage current, stability parameters which include the read, write, and hold margin along with the effect of variation of the gate length on the stability. The cell proposed in Figure 2 and Figure 3 has been simulated using FinFET $20 \mathrm{~nm}$ technology. The cells have been proposed for subthreshold conduction and the supply voltage has range has been kept in the range of $0.1 \mathrm{~V}$ to $0.4 \mathrm{~V}$. In this paper, the leakage current has been analyzed and calculated along with the stability of the cell with read margin, write margin \& hold margin have been determined and compared using the conventional SRAM cells. The simulation has been done using HSPICE at 20nm FinFET technology. 


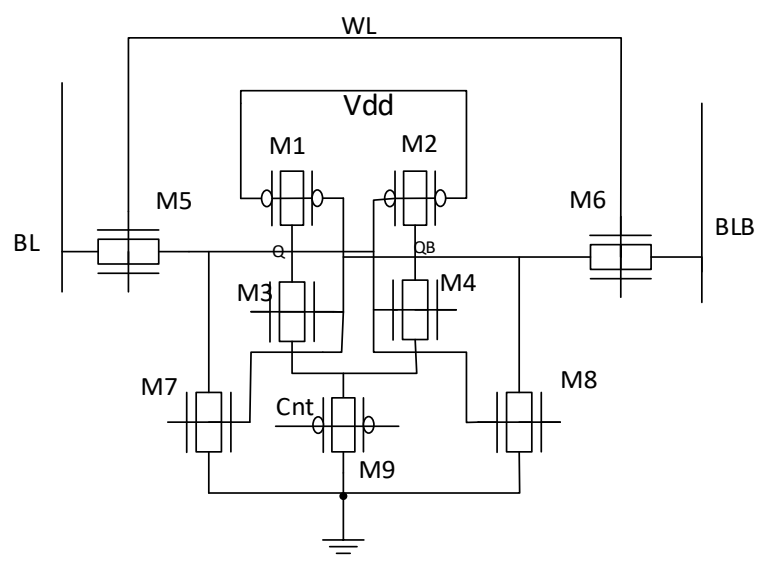

Figure 3. 9T FinFET improved SRAM cell

\subsection{Leakage power dissipation}

Leakage power dissipation is one of the key concern areas in handheld devices and all the handheld devices consist of SRAMs, so it will be a major concern for the memories like SRAM. Leakage current is measured majorly when memory remains idle, in cache memory leakage power is of major concern to focus on. The static power dissipation is calculated for both the proposed cells. The Static Power Dissipation for the proposed 9T SRAM cell is $1.5 \mathrm{nW}$ and for 9T FinFET, the improved SRAM cell was $1.1 \mathrm{nW}$ which was an improvement of 1.36x. Figure 4 shows the leakage current of the 9T SRAM and 9T improved SRAM cell at a subthreshold voltage of $0.4 \mathrm{~V}$. The leakage current measured is 77nA for 9T FinFET SRAM Cell and $69 n A$ of the proposed FinFET SRAM Table.

\subsection{Stability factors}

Static Noise Margin SNM is the key parameter in accessing the stability of the cell. It can be determined using the butterfly curve or $\mathrm{N}$ curves [20], [21]. There are three types of stability parameters used to find for an SRAM cell: read margin (RM), write margin (WM) \& hold margin (HM).

\subsubsection{Write margin}

The potential difference of the two-bit line levels which is responsible for flipping the data is defined as WM. The write-ability of the SRAM cell is evaluated by WM [22]. Figure 5 shows the WM for the three FinFET SRAM cells. It has been observed that the 9T improved SRAM FinFET cell has a better WM than the conventional 6T SRAM cell and proposed 9T SRAM cells. The write margin for the proposed 9T SRAM cell is $177.4 \mathrm{~V}$ and for the 9T improved SRAM cell has $264 \mathrm{mV}$ at $0.4 \mathrm{~V}$. The improved 9T SRAM cell shows an improvement of $1.49 \mathrm{x}$ over 9T SRAM at subthreshold voltage.

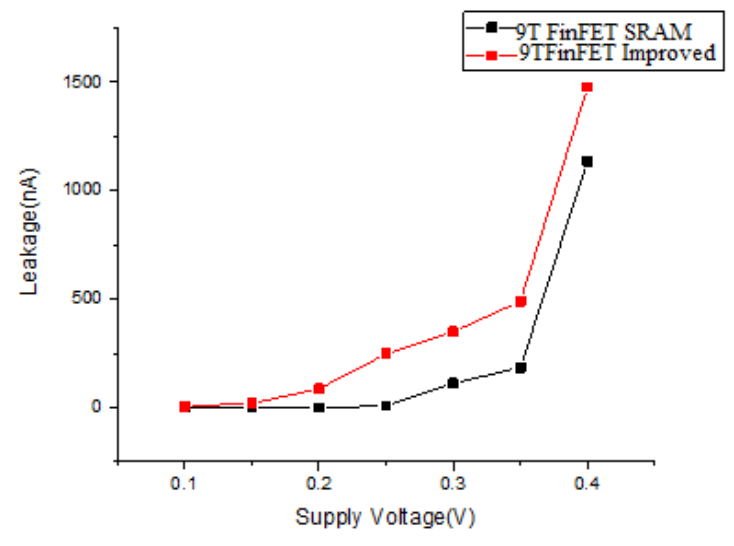

Figure 4. Leakage current of the various SRAM cells

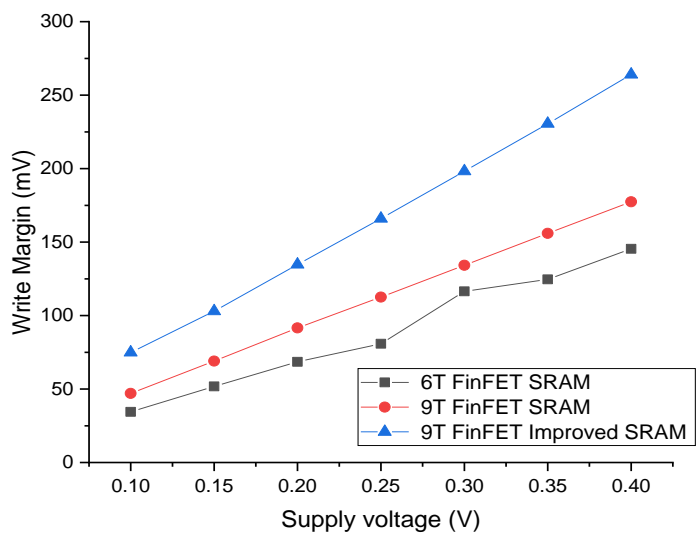

Figure 5. Write the margin of the various SRAM cells 


\subsubsection{Read margin}

Read margin is used to determine the read stability. SRAM cells are most affected by noise during read operation only as read failure is a common issue in most of the SRAM cells. The read stability for an SRAM cell can be determined by RM. During a read operation, a voltage divider is formed between the access transistor and pull-down transistor and so, the node which was at $0 \mathrm{~V}$ has raised to some positive voltage. A small voltage can change the stored value so RSNM is very much prone to noise and thus important [23]. Seevinck et al. [24] the noise margin is calculated using the butterfly curve in which it is said that the side length of the biggest square which can be embedded in the two lobes of the curve determines the SNM.

Figure 6 shows the variation of RM of 6T SRAM,9T SRAM \& 9T improved SRAM cell. The RM for the 9T improved SRAM cell is $170 \mathrm{mV}$ and for the 9T SRAM cell is $125 \mathrm{mV}$ at $0.4 \mathrm{~V}$. At $0.4 \mathrm{~V}$ supply voltage, 9T improved SRAM cell has $1.36 \mathrm{x}$ times read margin than 9T SRAM cell. The 9T improved SRAM cell proves to be better in read operation also.

\subsubsection{Hold margin}

A cell in its idle state is considered to hold the data, Hold SNM is a metric used to measure how well the cell has held the data. Hold SNM is decreased if the supply voltage is reduced, "Data retention voltage (DRV) is the minimum voltage at which the cell can continue to hold its state" [25].

The hold stability in terms of the HM in the subthreshold operations has been calculated for the SRAM cell for three cells 6T, 9T SRAM cell, and 9T improved SRAM cell as shown in Figure 7 and it can be noticed that 9T improved SRAM cell has better hold the margin as compared to the other two cells. AT $0.4 \mathrm{~V}$ the hold margin of the 9T FinFET SRAM cell is $264 \mathrm{mV}$ while for the 9T FinFET cell is $177.5 \mathrm{mV}$ and for the 6T SRAM cell, it is $144 \mathrm{mV}$. There is an improvement in the hold margin by $1.25 \mathrm{X}$ from the conventional 6T SRAM cell.

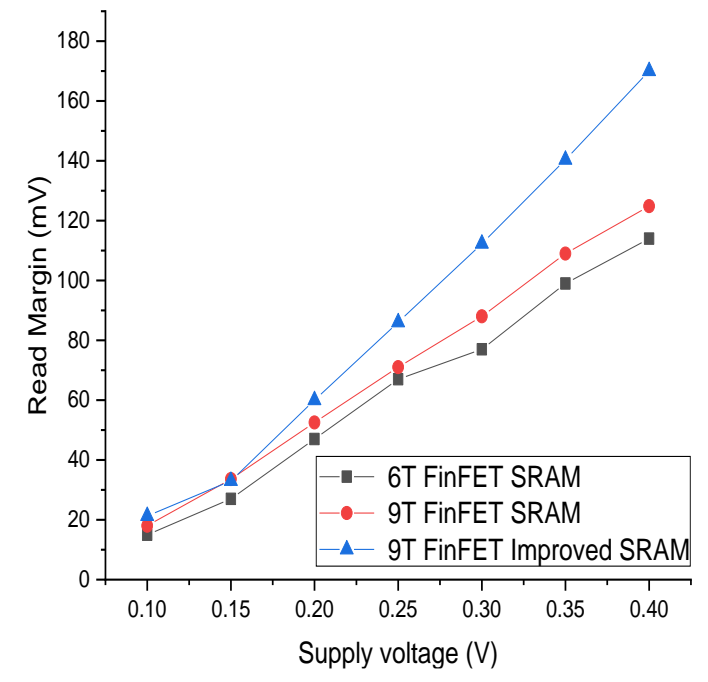

Figure 6. Read margin of the various SRAM cells

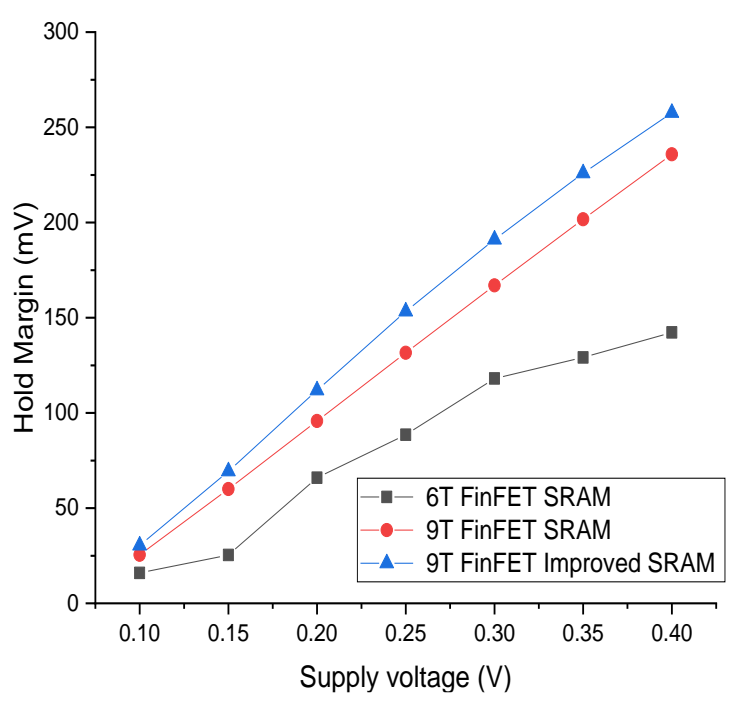

Figure 7. Hold margin of the various SRAM cells

\subsection{Effect of gate length over the RM}

The previous section discussed the stability factors concerning the variation of the supply voltage. Effect of stability factors i.e. RM, WM, and HM by varying the gate length has been found for 9T SRAM cell \& 9T improved SRAM cell. The effect of variation of gate length on WSNM \& gate length was negligible in both the cells and the effect on RSNM is considerable as shown in Figure 8. RSNM of the gate can be increased up to $20 \%$ if gate length is increased by $10 \%$ in 9T improved SRAM cell. The variation in RSNN is more in 9T improved SRAM cell as compared to a 9T SRAM cell. 


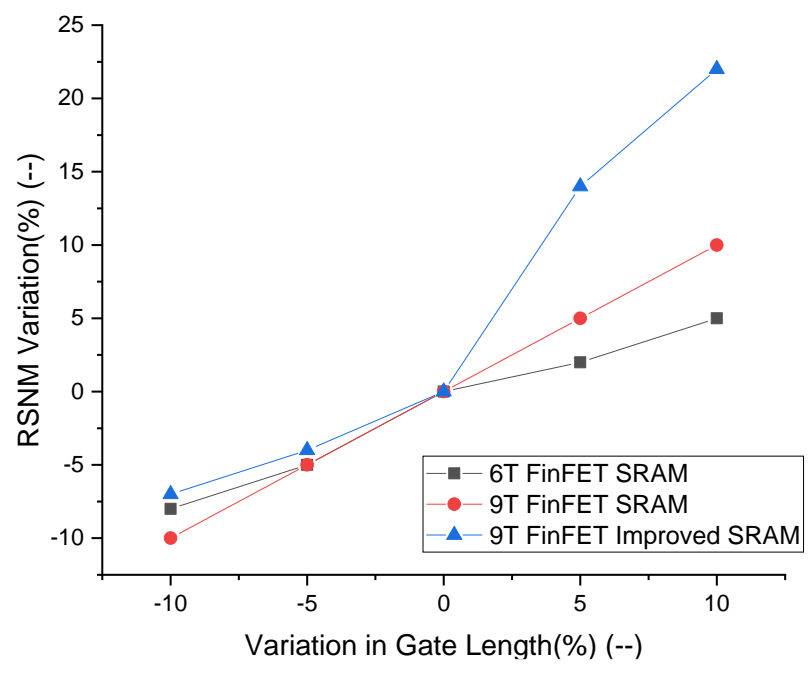

Figure 8. RSNM variation wrt. to gate length

\section{CONCLUSION}

In this paper, two different SRAM cells have been projected using FinFET for better stability and less leakage current. The leakage power and stability are found better in a 9T improved SRM cell as compared to a 9T SRAM cell. Both the cells have shown better overall better performance from 6T SRAM cells. Hold margin improved by $1.2 \mathrm{x}$, read margin improved by $1.36 \mathrm{x}$, and write margin improved by $1.49 \mathrm{x}$. The effect of variation of gate length has also been found and seen that increasing the gate length will increase the RSNM. The future scope of this work is an SRAM array that can be designed and simulated for various operations. The speed can be optimized which is the future scope of this work. The only limitation of 9T improved SRAM cell is that since one extra PMOS transistor is used it can increase the area.

\section{ACKNOWLEDGEMENT}

We would like to thank our organization who has given us this opportunity to do this research work. The authors are thankful to Manipal University Jaipur and to the King Khalid University, Kingdom of Saudi Arabia for the DSR financial support in publication of this research work under the grant number RGP1/224/42.

\section{REFERENCES}

[1] N. Verma, J. Kwong and A. P. Chandrakasan, "Nanometer MOSFET Variation in Minimum Energy Subthreshold Circuits," in IEEE Transactions on Electron Devices, vol. 55, no. 1, pp. 163-174, Jan. 2008, doi: 10.1109/TED.2007.911352.

[2] T. Cakici, K. Kim and K. Roy, "FinFET Based SRAM Design for Low Standby Power Applications," 8th International Symposium on Quality Electronic Design (ISQED'07), 2007, pp. 127-132, doi: 10.1109/ISQED.2007.76.

[3] Y. S. Chauhan et al., "FinFET modeling for IC simulation and design using the BSIM-CMG standard," Elsevier Science, 2015.

[4] J. H. Lee, "Bulk FinFETs: Design at $14 \mathrm{~nm}$ Node and Key Characteristics, Nanodevices and circuit techniques for low energy application and energy harvesting," KAIST Research series, pp. 33-64, 2016, doi: 10.1007/978-94-0179990-4_2.

[5] B. Ebrahimi, A. Afzalikusha and H. Mahmoodi, "Robust FinFET SRAM design based on dynamic back gate voltage adjustment," Microelectronics Reliability, vol. 5, pp. 2604-2612, 2004, doi: 10.1016/j.microrel.2014.04.015.

[6] N. K. Jha and D. Chen, "Nanoelectronic circuit design," Springer Science, pp. 50-70, 2011, doi: 10.1007/978-14419-7609-3.

[7] Z. Liu and V. Kursun, "Characterization of a Novel Nine-Transistor SRAM Cell," in IEEE Transactions on Very Large Scale Integration (VLSI) Systems, vol. 16, no. 4, pp. 488-492, April 2008, doi: 10.1109/TVLSI.2007.915499.

[8] S. Pal and A. Islam, "Variation Tolerant Differential 8T SRAM Cell for Ultralow Power Applications," in IEEE Transactions on Computer-Aided Design of Integrated Circuits and Systems, vol. 35, no. 4, pp. 549-558, April 2016, doi: 10.1109/TCAD.2015.2474408. 
[9] S. K. Gupta, J. P. Kulkarni and K. Roy, "Tri-Mode Independent Gate FinFET-Based SRAM With Pass-Gate Feedback: Technology-Circuit Co-Design for Enhanced Cell Stability," in IEEE Transactions on Electron Devices, vol. 60, no. 11, pp. 3696-3704, Nov. 2013, doi: 10.1109/TED.2013.2283235.

[10] S. M. Salahuddin and M. Chan, "Eight-FinFET Fully Differential SRAM Cell With Enhanced Read and Write Voltage Margins," in IEEE Transactions on Electron Devices, vol. 62, no. 6, pp. 2014-2021, June 2015, doi: 10.1109/TED.2015.2424376.

[11] S. A. Tawkif and V. Kursun, "Robust FinFET memory circuits with p-type data access transistors for higher integration density and reduced leakage power," Journal of Low Power Electronics, vol. 5, pp. 497-508, 2009, doi: 10.1166/jolpe.2009.1048.

[12] S. Birla, "Subthreshold FinFET SRAM cell at 20nm Technology with improved Stability \& Leakage Power," Indian Journal of Science \& Technology, vol.10, no. 2, pp. 1-6, 2017, doi: 10.17485/ijst/2017/v10i3/110626.

[13] B. Zeinali, J. K. Madsen, P. Raghavan and F. Moradi, "Sub-Threshold SRAM Design in 14 Nm FinFET Technology with Improved Access Time and Leakage Power," 2015 IEEE Computer Society Annual Symposium on VLSI, 2015, pp. 74-79, doi: 10.1109/ISVLSI.2015.73.

[14] Limachia, Mitesh, Rajesh Thakker and Nikhil Kothari. "A near-threshold 10t differential SRAM cell with high read and write margins for tri-gated Finfet technology," Integration, vol. 61, pp. 125-137, 2018, doi: 10.1016/j.vlsi.2017.11.009.

[15] Manju, I. and A. Senthil Kumar. "A $22 \mathrm{~nm}$ FinFET based 6T-SRAM cell design with scaled supply voltage for increased read access time," Analog Integrated Circuits and Signal Processing, vol. 84, no. 1, pp : 119-126, 2018, doi: 10.1007/s10470-015-0547-6.

[16] J. P. Kulkarni, Keejong Kim, S. P. Park and K. Roy, "Process variation tolerant SRAM array for ultra low voltage applications," 2008 45th ACM/IEEE Design Automation Conference, 2008, pp. 108-113, doi: $10.1145 / 1391469.1391498$.

[17] C. Duari, S. Birla and A. K. Singh. "A dual port 8T SRAM cell using FinFET \& CMOS logic for leakage reduction and enhanced read \& write stability," Journal of Integrated Circuits and Systems, vol. 15, no. 2, pp: 1-7, 2020, doi: 10.29292/jics.v15i2.140.

[18] Shilpi Birla, "Variability aware FinFET SRAM cell with improved stability and power for low power applications." Circuit World, 2019, doi: 10.1108/CW-12-2018-0098.

[19] Salahuddin, Shairfe Muhammad, Volkan Kursun and Hailong Jiao, "Finfet sram cells with asymmetrical bitline access transistors for enhanced read stability." Transactions on Electrical and Electronic Materials, vol.16, no. 6, pp: 293-302, 2015, doi: 10.4313/TEEM.2015.16.6.293.

[20] B. H. Calhoun and A. P. Chandrakasan, "Static noise margin variation for sub-threshold SRAM in 65-nm CMOS," in IEEE Journal of Solid-State Circuits, vol. 41, no. 7, pp. 1673-1679, July 2006, doi: 10.1109/JSSC.2006.873215.

[21] E. Grossar, M. Stucchi, K. Maex and W. Dehaene, "Read Stability and Write-Ability Analysis of SRAM Cells for Nanometer Technologies," in IEEE Journal of Solid-State Circuits, vol. 41, no. 11, pp. 2577-2588, Nov. 2006, doi: 10.1109/JSSC.2006.883344.

[22] A. Islam and M. Hasan, "Leakage Characterization of 10T SRAM Cell," in IEEE Transactions on Electron Devices, vol. 59, no. 3, pp. 631-638, March 2012, doi: 10.1109/TED.2011.2181387.

[23] A. Islam and M. Hasan, "A technique to mitigate the impact of the process, voltage and temperature variations on design metrics of SRAM cell," Microelectronics Reliability, vol. 52, no. 2, pp. 405-411, 2012, doi: 10.1016/j.microrel.2011.09.034.

[24] E. Seevinck, F. J. List and J. Lohstroh, "Static-noise margin analysis of MOS SRAM cells," in IEEE Journal of Solid-State Circuits, vol. 22, no. 5, pp. 748-754, Oct. 1987, doi: 10.1109/JSSC.1987.1052809.

[25] M. Qazi, M. Sinangil and A. Chandrakasan, "Challenges and Directions for Low-Voltage SRAM," in IEEE Design \& Test of Computers, vol. 28, no. 1, pp. 32-43, Jan.-Feb. 2011, doi: 10.1109/MDT.2010.115.

\section{BIOGRAPHIES OF AUTHORS}

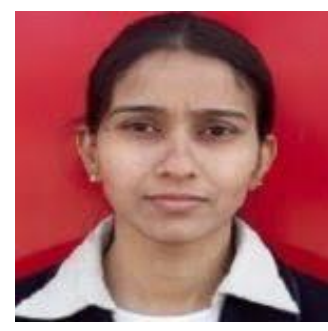

Shilpi Birla working as an Associate Professor at Manipal University Jaipur. She did her Ph.D. in VLSI. Her research interests are Low Power VLSI Design, Memory Circuits, Digital VLSI Circuits, Nanodevices. She has authored several research papers in journals of repute and International Conferences. She is the reviewer of several journals, conferences. She has guided many M. Tech Students and guiding Ph.D. Students. She is a senior member of IEEE. 


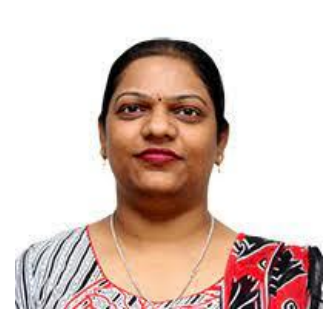

Neha Singh is working as an Assistant Professor at Manipal University Jaipur. She earned her $\mathrm{Ph}$.D. from Manipal University Jaipur She has been teaching. She has been in research in Image Processing and VLSI. She has authored several papers in International Journals and Conferences.

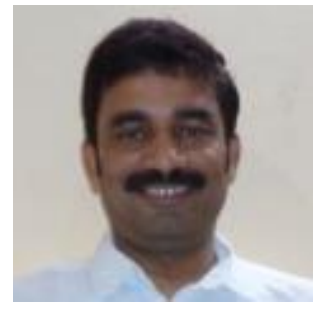

Neeraj Kumar Shukla is currently working as an Associate Professor at King Khalid University. He has academic, research, and industry experience of more than 20 years. He did his Ph.D. in VLSI Design; He has authored more than 100 research publications in journals of repute and conferences. He has mentored several M. Tech students and Ph.D. students.

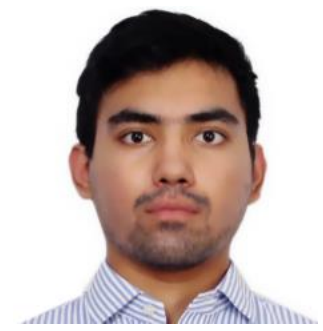

Sidharth Sharma is currently working as a Software Development Engineer at ServiceNow Ireland. Prior to this he was doing research for Intel R\&D Ireland on Deep Neural Network Accelerators. 